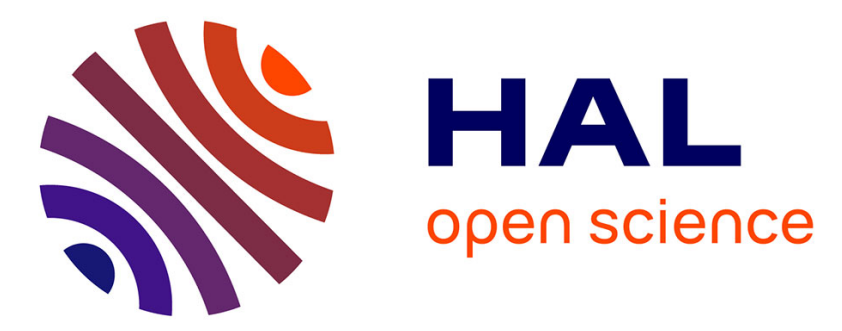

\title{
Influence de sollicitations hydriques et mécaniques complexes sur le comportement d'un sol gonflant compacté
}

Olivier Cuisinier, Farimah Masrouri

\section{- To cite this version:}

Olivier Cuisinier, Farimah Masrouri. Influence de sollicitations hydriques et mécaniques complexes sur le comportement d'un sol gonflant compacté. Canadian Geotechnical Journal, 2005, 42 (3), pp.731741. 10.1139/t05-007 . hal-01768462

\section{HAL Id: hal-01768462 \\ https://hal.science/hal-01768462}

Submitted on 17 Apr 2018

HAL is a multi-disciplinary open access archive for the deposit and dissemination of scientific research documents, whether they are published or not. The documents may come from teaching and research institutions in France or abroad, or from public or private research centers.
L'archive ouverte pluridisciplinaire HAL, est destinée au dépôt et à la diffusion de documents scientifiques de niveau recherche, publiés ou non, émanant des établissements d'enseignement et de recherche français ou étrangers, des laboratoires publics ou privés. 
INFLUENCE DE SOLLICITATIONS HYDRIQUES ET MÉCANIQUES

COMPLEXES SUR LE COMPORTEMENT D'UN SOL GONFLANT

COMPACTÉ

\section{Olivier CUISINIER ${ }^{1,2}$ et Farimah MASROURI ${ }^{1, *}$}

1 : Laboratoire Environnement Géomécanique et Ouvrages

École Nationale Supérieure de Géologie

Institut National Polytechnique de Lorraine

Rue du Doyen Marcel Roubault - BP 40

54501 Vandœuvre-lès-Nancy - France

2: Adresse actuelle :

Laboratoire Central des Ponts et Chaussées

Route de Bouaye - BP 4129

44341 Bouguenais - France

* Auteur auquel doit être adressée toute correspondance.

Tél. : +33 383596304

Fax : +33 83596300

Farimah.Masrouri@ensg.inpl-nancy.fr 


\section{Influence de sollicitations hydriques et mécaniques complexes sur le comportement d'un sol gonflant compacté}

\section{Olivier CUISINIER et Farimah MASROURI}

\section{RÉSUMÉ}

L’objectif de cet article est de présenter une étude sur les déformations d'un sol gonflant provoquées par des sollicitations hydriques et/ou mécaniques complexes ainsi que leurs conséquences sur sa compressibilité notamment. Dans la première partie de l'article, les dispositifs expérimentaux à succion contrôlée employés (méthodes osmotique et solutions salines) pour conduire l'ensemble des essais sont décrits brièvement. Les essais ont été réalisés dans la gamme des succions comprises entre 0 et $40 \mathrm{MPa}$. Les conséquences d’une sollicitation hydrique monotone (humidification) conduite sous différentes contraintes ont d’abord été caractérisées. Il est apparu que dans ces conditions d’essai, les déformations dépendent du chemin de contrainte contrairement à la pente de compression plastique $\lambda(\mathrm{s})$ qui ne dépend que de la succion imposée durant le chargement mécanique. Une deuxième série d'essais a permis de montrer l'influence du chemin de contrainte sur les déformations ainsi que la pente $\lambda(s)$ si une succion supérieure à la succion initiale des éprouvettes est imposée au cours de l'essai (dessiccation). En conclusion, la valeur de la pente $\lambda$ (s) dépend de la succion sous laquelle est effectué le chargement mécanique mais aussi de la plus grande succion connue par le matériau au cours de son « histoire ».

\section{MOTS CLÉS}

Sol compacté ; succion ; cycle hydrique ; comportement hydromécanique ; sol gonflant. 


\section{Influence of complex hydromechanical loadings on the behaviour of a compacted expansive soil}

\section{Olivier CUISINIER and Farimah MASROURI}

\section{ABSTRACT}

The main objective of the paper is to present a study on compacted expansive soil strains generated by complex hydric or mechanical loadings and their consequences on tested soil compressibility. In the first part of the paper, the suction controlled testing devices are described (osmotic and salt solutions methods). All the presented tests were performed in the range of suctions comprised between 0 and $40 \mathrm{MPa}$. The consequences of the strains induced by wetting as a function of the applied mechanical stress were analyzed. The results showed that, under these conditions, strain depends on the followed hydraulic path whereas the slope of the plastic compression line $\lambda(\mathrm{s})$ was only affected by the suction applied during the mechanical loading. The second test series demonstrated that the stress path influenced both deformation and slope $\lambda(s)$ if suction higher than the initial sample suction was imposed during the test. As a conclusion, it could be stated that the slope $\lambda(\mathrm{s})$ depends on the suction applied during a mechanical loading and on the maximum suction experienced by an expansive material during its past "history”.

\section{KEYWORDS}

Compacted soil; suction; hydric cycle; hydromechanical behaviour; expansive soil. 


\section{INTRODUCTION}

Les matériaux gonflants compactés ont pendant longtemps été étudiés en raison des désordres générés par leur présence (e.g. Chen 1975 ; Philipponat 1991 ; Vandangeon 1992). Leurs propriétés particulières, faible perméabilité et capacité de rétention notamment, en font cependant des matériaux très intéressants dans de nombreuses applications. Ils sont ainsi employés en géotechnique de l'environnement pour la construction de sites de stockage de déchets ménagers dont Koch (2002) a présenté une synthèse. Actuellement, leur utilisation est envisagée pour la construction de barrières ouvragées destinées à confiner des colis de déchets nucléaires stockés en profondeur (e.g. Chapman et Tassoni 1985 ; Gens et Olivella 2001). Dans tous les cas, il est nécessaire d'assurer la stabilité des ouvrages réalisés sur plusieurs siècles. Durant ce laps de temps, il est probable que ces matériaux seront soumis, entre autres, à des sollicitations de type humidification/dessiccation sous différentes conditions mécaniques. Il est donc indispensable de connaître les conséquences de ces sollicitations sur les propriétés hydromécaniques, notamment la compressibilité, de ces matériaux.

Pour étudier les phénomènes en jeu, l'utilisation de deux variables indépendantes, définies à partir de la contrainte totale $\sigma$, de la pression de l'air $u_{a}$, et de la pression de l'eau $u_{w}$, a été proposée (Coleman 1962 ; Matyas et Radakrishna 1968 ; Fredlund et Morgenstern 1977). Il s'agit de la contrainte nette $\sigma^{*}=\sigma-\mathrm{u}_{\mathrm{a}}$ et de la succion $\mathrm{s}=\mathrm{u}_{\mathrm{a}}-\mathrm{u}_{\mathrm{w}}$.

Dans ce contexte, les matériaux gonflants compactés ont fait l’objet de nombreuses études, notamment pour déterminer la sensibilité de leurs caractéristiques mécaniques en fonction de leur succion (e.g. Push 1982 ; Gens et Alonso 1992 ; Guiras-Skandaji 1996 ; Romero et al. 1999 ; Cui et al. 2002). Ces travaux ont, entre autres, permis de mettre en évidence la forte dépendance de leur compressibilité vis-à-vis de la succion s, ainsi que le rôle de leur structure interne sur leur comportement mécanique, ce qui a abouti au développement de modèles de comportement (e.g. Alonso et al. 1999). Cependant, la plupart de ces conclusions ont été 
déduites d'essais n’impliquant que des sollicitations hydriques monotones (humidification ou dessiccation) suivies d'un chargement mécanique. Or, de nombreux auteurs, travaillant sur une grande variété de matériaux mais sans dispositif d’imposition de succion, ont montré que la succession de cycles hydriques induisait l'accumulation de déformations irréversibles associées à des modifications profondes de la structure interne de ce type de sol (Tessier 1984 ; Osipov et al. 1987 ; Dif et Bluemel 1991 ; Day 1994). Des essais de même type comportant des cycles hydriques réalisés à succion contrôlée sur d'autres sols gonflants ont permis à Alonso et al. (1995) de même que Kato et Kawai (2000) de souligner l'influence de la contrainte verticale appliquée pendant le cycle hydrique sur ce processus. Villar (1999), en travaillant sur l'argile de Boom, a montré que les déformations plastiques étaient d’autant plus faible que la contrainte appliquée lors du cycle hydrique était élevée, ces essais ayant été réalisés dans une large gamme de succions. De même, une étude plus systématique de Lloret et al. (2003) a mis en évidence la dépendance des déformations vis-à-vis du chemin de contrainte suivi dans le cas d'une bentonite fortement compactée.

Jusqu’à présent, à la connaissance des auteurs de cet article, les conséquences de ces phénomènes sur la compressibilité n’ont fait l’objet que de peu d'investigations. Sharma (1998), cité par Gallipoli et al. (2003), a mis en évidence une dépendance significative de la pente de compression plastique $\lambda(\mathrm{s})$, définie par la relation $\lambda(\mathrm{s})=\Delta \mathrm{e} / \Delta \ln \left(\sigma_{\mathrm{v}}^{*}\right)$ avec e l'indice des vides, $\mathrm{s}$ la succion et $\sigma_{\mathrm{v}}^{*}$ la contrainte verticale nette, vis-à-vis du chemin de contrainte suivi lors d'un essai en travaillant sur un mélange compacté de kaolin et de bentonite. Les résultats de cet auteur tendent à montrer que la compressibilité d'un matériau gonflant est modifiée par l'imposition d'un cycle hydrique d'humidification/dessiccation. Dans ce contexte, il apparaît important de caractériser ces phénomènes d'une manière plus systématique dans une large gamme de succion (0 à plusieurs dizaines de MPa). Une étude de l’influence de sollicitations hydromécaniques complexes sur le comportement mécanique 
d’un matériau gonflant a donc été entreprise. L’objectif de ce travail est de préciser sous quelles conditions le chemin de contrainte peut influencer sur les déformations et la compressibilité d’un sol gonflant compacté.

Pour réaliser cette étude, deux types d’appareils œdométriques à succion contrôlée ont été utilisés, leurs principes respectifs sont brièvement décrits dans le premier point de cet article. L'étude de sollicitations hydriques monotones sous différentes conditions mécaniques a ensuite été réalisée et les principaux résultats sont exposés dans la seconde partie. Le troisième point de l'article se concentre sur l'influence de sollicitations hydriques cycliques réalisées entre des succions de 8,5 et 38,9 MPa sous différentes conditions mécaniques. La dépendance des déformations et des paramètres mécaniques vis-à-vis du chemin de contrainte suivi est étudiée à chaque fois. Les principales conclusions de l'étude sont ensuite exposées.

\section{DISPOSITIFS EXPÉRIMENTAUX}

Pour réaliser les essais présentés dans cet article, deux types d’œdomètre à succion contrôlée ont été employés : il s’agit d'œdomètres osmotiques et d'œdomètres à solutions salines. Ces appareils ont fait l’objet d’une description détaillée par Cuisinier et Masrouri (2003, 2004a) et seuls leurs principes généraux seront rappelés ici.

\section{Edomètre à succion contrôlée par la méthode osmotique}

Le principe de la méthode est de mettre en contact une éprouvette de sol et une solution de macromolécules en insérant entre les deux une membrane semi-perméable (Zur 1966). Celleci empêche le passage des macromolécules de la solution vers l'éprouvette mais elle permet les échanges d'eau. Les mouvements d'eau, et donc la succion, sont contrôlés par le phénomène d’osmose : plus la concentration en macromolécules est élevée, plus la succion imposée est forte. La macromolécule utilisée classiquement est le polyéthylène glycol (PEG) avec différents poids moléculaires. Le schéma de principe de l'œdomètre osmotique utilisé est 
donné sur la Figure 1, la base de l’œdomètre permettant de faire circuler la solution osmotique au contact de l'éprouvette. La relation entre la concentration en macromolécules et la succion a été caractérisée par différents auteurs (Williams et Shaykewich 1969 ; Delage et al. 1998 ; Cuisinier et Masrouri 2004a). En l'état actuel et avec le dispositif employé, cette méthode a permis d'imposer des succions comprises entre 0 et 8,5 MPa. Il est possible d'imposer plusieurs succions de manière successive au cours d'un essai. Pour effectuer le changement de succion, la circulation est arrêtée quelques minutes et un nouveau réservoir, contenant une solution osmotique à une concentration différente, est mis en place à la place du premier. La circulation est ensuite remise en route. La concentration du réservoir retiré est ensuite déterminée, ce qui permet de contrôler la succion imposée. Dans le cas d’un œdomètre osmotique, il faut environ 10 jours pour atteindre la stabilisation des déformations et des échanges hydriques lors d’un palier hydrique (Cuisinier 2002).

\section{Edomètre à succion contrôlée par solutions salines saturées}

Le principe de ce type d'œdomètre, initialement décrit par Esteban (1988) cité par Villar et Martín (1996), est d'enfermer un système équivalent à une cellule œdométrique classique dans une enceinte dont l'humidité relative est maintenue constante grâce à une solution saline saturée. L’humidité relative Hr, est reliée à la succion s, par la loi de Kelvin :

$$
\mathrm{s}=-\gamma_{\mathrm{w}} \frac{\mathrm{R} \mathrm{T}}{\mathrm{Mg}} \ln (\mathrm{Hr})
$$

avec $\gamma_{\mathrm{w}}$ le poids volumique de l'eau $\left(9,81 \mathrm{kN} \cdot \mathrm{m}^{-3}\right)$, g l'accélération de la pesanteur, $\mathrm{M}$ la masse molaire de l'eau $\left(18 \times 10^{-3} \mathrm{~kg} \cdot \mathrm{mol}^{-1}\right)$, R la constante des gaz parfaits $\left(8,31 \mathrm{~J}^{\mathrm{mol}}{ }^{-1} \cdot{ }^{\circ} \mathrm{K}^{-1}\right)$ et $\mathrm{T}$ la température absolue $\left({ }^{\circ} \mathrm{K}\right)$. Deux nouveaux appareils œdométriques avec deux capacités d’application de contrainte verticale maximale différentes (1 200 ou $20000 \mathrm{kPa}$ ), ont été développés sur le même principe (Figure 2). L’utilisation de plusieurs solutions salines saturées permet d'imposer différentes succions, la valeur de l'humidité relative imposée par 
chaque sel est disponible dans Lide (2002). Les conditions d’utilisation détaillées de cette méthode sont exposées dans Delage et al. (1998) et Cuisinier (2002). Elle permet d'imposer des succions allant de 8,5 jusqu'à plusieurs centaines de MPa. Les solutions salines employées dans cette étude sont données dans le Tableau 1. Dans le cas de ces appareils il faut environ 20 à 30 jours pour atteindre la stabilisation des déformations de l'éprouvette lors d’un palier hydrique (Cuisinier 2002).

\section{MATÉRIAU ÉTUDIÉ ET PRÉPARATION DES ÉPROUVETTES}

Le matériau étudié est un mélange de $40 \%$ d'un limon et de $60 \%$ de bentonite calcique d’appellation commerciale FVO (Société française des bentonites et dérivés). Les différentes caractéristiques géotechniques de ces matériaux et du mélange sont données dans le Tableau 2. Le limon et la bentonite, initialement sous forme de poudres tamisées à $400 \mu \mathrm{m}$, sont mélangés puis humidifiés à une teneur en eau massique de $15 \%$. Cette valeur, estimée comme proche de la limite de retrait du mélange, a été sélectionnée de manière à limiter la rétraction de l'éprouvette lors de l'imposition d'une dessiccation, c'est-à-dire d'une augmentation de succion. Le mélange humide a ensuite été conservé au moins une semaine dans un récipient hermétique. Après cette période, les éprouvettes ont été préparées par compactage statique (vitesse de $1,14 \mathrm{~mm} \cdot \mathrm{min}^{-1}$ ) sous une contrainte verticale de $1000 \mathrm{kPa}$

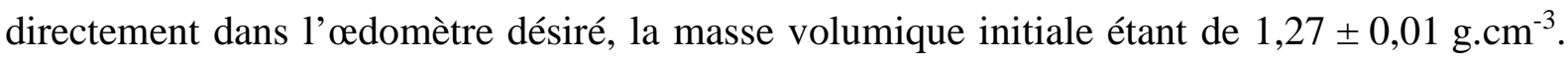
La hauteur initiale des éprouvettes est de $1 \pm 0,05 \mathrm{~cm}$ quel que soit l'appareil employé, leur diamètre vaut $7,4 \mathrm{~cm}$ dans les œdomètres à solutions salines et $7 \mathrm{~cm}$ dans les œdomètres osmotiques. La succion totale initiale a été mesurée par la technique du papier filtre (ASTM 1995a). Elle est comprise entre 20 et $25 \mathrm{MPa}$.

Pour caractériser les propriétés de gonflement du matériau lorsque la succion de l'éprouvette est ramenée à $0 \mathrm{MPa}$, deux paramètres ont été déterminés. Il s’agit du potentiel de 
gonflement, qui est le rapport de la variation de hauteur de l'éprouvette produite par l'humidification sur sa hauteur initiale, et la pression de gonflement, qui correspond ici à la pression nécessaire pour éliminer la variation de hauteur produite lors de l’humidification ; ces grandeurs, déterminées par la méthode du gonflement libre (ASTM 1995b), sont de 17 \% et de $250 \mathrm{kPa}$ respectivement.

Cette masse volumique sèche initiale relativement basse a été choisie de manière à obtenir un matériau compacté présentant initialement une double structure marquée : les éprouvettes comprennent des agrégats entre lesquels existent des macropores. Ce type de structure a été observé à la fois dans les sols naturels (e.g. Collins et McGown 1974) et sur les matériaux gonflants compactés (Push 1982). L’objectif était de faciliter la mise en évidence de l'influence éventuelle des sollicitations hydriques complexes sur le comportement d'un tel matériau, notamment sur sa compressibilité. L’observation d’éprouvettes compactées du matériau employé à partir de l’observation de lames minces a permis de confirmer que les éprouvettes présentaient bien initialement une double structure après la phase de compactage (Cuisinier et Masrouri 2004b).

\section{INFLUENCE D'UNE SOLLICITATION HYDRIQUE MONOTONE SUR LE COMPORTEMENT MÉCANIQUE}

Dans cette partie, deux séries d'essais sont présentées, leur objectif est d’évaluer les conséquences sur le comportement mécanique d’une modification monotone de succion en fonction des conditions mécaniques sous laquelle elle est entreprise. Ces données complètent l'étude réalisée précédemment sur le même matériau qui avait pour but de caractériser la variation des propriétés hydromécaniques du même matériau dans une gamme de succion comprise entre 0 et $300 \mathrm{MPa}$ environ (Cuisinier et Masrouri 2004a).

Effet d'un changement de succion à volume constant 
Lorsqu'un sol gonflant est humidifié à volume constant, il développe une pression qui peut être évaluée par la méthode C de la procédure ASTM D 4546 - 90. Dans le cadre de cette étude, cette pression a été déterminée par la méthode d’humidification à volume constant (essai $V_{c}$ ) en utilisant un dispositif expérimental spécialement dédié. Il utilise une base d’appareil œdométrique osmotique identique à celle indiquée sur la Figure 2, le piston étant remplacé par un dispositif fixe qui permet d'empêcher le gonflement de l'éprouvette lors de l'humidification. Ce dispositif comprend un capteur de pression qui permet l'enregistrement de la pression exercée par le matériau au cours de son humidification.

La variation de la pression exercée par le matériau en fonction du temps écoulé depuis l'humidification est donnée sur la Figure 3 et les caractéristiques de l'éprouvette sont indiquées dans le Tableau 3 qui donne la teneur en eau massique initiale $\mathrm{w}_{\mathrm{i}}$, la teneur en eau massique finale $\mathrm{w}_{\mathrm{f}}$, la masse volumique sec initiale $\rho_{\mathrm{di}}$, le degré de saturation initial $\mathrm{Sr}_{\mathrm{i}}$, et le degré de saturation final $\mathrm{Sr}_{\mathrm{f}}$ de chaque éprouvette. Les résultats de cet essai montrent que la pression exercée passe par un maximum, environ $58 \mathrm{kPa}$, avant de décroître pour atteindre une valeur finale de $49 \mathrm{kPa}$ au cours du temps. Ces observations sont similaires à celles qui avaient été obtenues par différents auteurs (e.g. Brackley 1973 ; Push 1982 ; Alonso et al. 1999). La décroissance de la pression après le pic peut être expliquée par une plastification de l'éprouvette au cours de l'humidification.

Afin de compléter ce résultat, une série d'essais d'humidification sous différentes charges verticales a été entreprise.

\section{Effets d'une humidification sous charge constante}

Lorsqu'un sol initialement non saturé est humidifié (réduction de succion) sous charge, une réduction de l'indice des vides peut se produire lors de la mise en eau : c'est le phénomène 
d'effondrement (e.g. Barden et al. 1969 ; Barrera et al. 2002). Plusieurs essais de ce type ont été réalisés avec pour objectif la détermination du comportement mécanique du matériau après la phase d'effondrement (Figure 4).

La première étape de ces essais a consisté à appliquer la contrainte verticale sous laquelle l'humidification devait être conduite. Les valeurs choisies sont 10, 100, 200 ou 800 kPa, ce qui correspond respectivement aux essais Hu1, Hu2, Hu3 et Hu4. Dans cette phase une succion de 20,5 MPa, voisine de la succion initiale des éprouvettes, a été appliquée dans le cas de l'essai Hu4 réalisé dans un œdomètre à solutions salines. Avec les appareils osmotiques, il n’a pas été possible durant cette phase d’imposer une succion voisine de la succion initiale des éprouvettes, celle-ci étant supérieure à la succion maximale imposable dans ce type d’œdomètre. Par souci de simplification nous avons cependant représenté les points A, B et C comme étant à une succion de 20,5 MPa sur la Figure 4. Au cours de la deuxième phase de l'essai (humidification sous charge), la succion des éprouvettes a été réduite en plusieurs étapes jusqu’à une succion voisine de 0 (cf. Figure 4). Dans le cas de l'essai Hu4, une seule étape de réduction de succion a pu être imposée, de la succion initiale vers 8,5 MPa, cette succion correspond en effet à la plus basse succion qu'il est possible d'imposer dans un appareil à solutions salines. Dans tous les essais, un chargement/déchargement mécanique a été réalisé après la phase d’humidification. Les caractéristiques initiales et finales des éprouvettes sont indiquées dans le Tableau 3.

La variation de volume de chaque éprouvette, exprimée comme le rapport entre la variation de hauteur et de la hauteur initiale, lors de la phase d'humidification en fonction de la succion appliquée est donné sur la Figure 5. L’éprouvette Hu1 gonfle jusqu’à une valeur de 17,2 \% lorsque s = $0 \mathrm{MPa}$. La variation de volume observée dans l'essai Hu2 est voisine de celle obtenue dans le cas de l'essai Hu1 jusqu'à une succion appliquée de 2 MPa. Cette éprouvette s’effondre cependant lors de l'imposition d’une succion inférieure à 2 MPa. Dans cet essai, 
les réductions de succion ultérieures ne provoquent pas de variation de volume supplémentaire. L’éprouvette Hu3 ne subit qu'un léger gonflement jusqu’à une succion imposée de $5 \mathrm{MPa}$, en deçà de cette succion un effondrement est obtenu, qui progresse jusqu’à une succion imposée de 0,5 MPa. Dans le cas de l’essai Hu4, réalisé dans un appareil à solutions salines, l'effondrement est obtenu entre 20,5 $\mathrm{MPa}$ et 8,5 $\mathrm{MPa}$. Ces données permettent donc de caractériser la relation entre la charge appliquée et la succion pour laquelle le matériau est susceptible de s'effondrer qui est d'autant plus élevée que la charge verticale appliquée est forte. De même, l'amplitude de l'effondrement augmente avec la charge appliquée. Ces résultats rejoignent les observations faites par Alonso et al. (1995).

Les résultats du cycle chargement/déchargement mécanique réalisé après la phase d’humidification dans chaque essai sont donnés sur la Figure 6. Dans chaque cas, la pente de compression plastique $\lambda(\mathrm{s})$ a été déterminée à partir du dernier palier de chargement mécanique. Deux cas sont à prendre en compte en fonction de la succion sous laquelle le chargement mécanique a été effectué. Les résultats des essais Hu1, Hu2 et Hu3 ont été comparés entre eux, la succion sous laquelle le chargement a été effectué étant nulle dans ces essais. Pour compléter le résultat de l'essai Hu4, où la succion appliquée lors du chargement est de 8,5 MPa, l'essai Ch1 a été réalisé. Le chemin de contrainte de cet essai correspond à l'imposition d'une succion de 8,5 MPa sous une contrainte verticale nette de $10 \mathrm{kPa}$ suivi par un chargement mécanique à succion constante (cf. Figure 4). Les caractéristiques de l'éprouvette sont données dans le Tableau 3, les courbes de compressibilité sur la Figure 6 et les paramètres mécaniques déterminés au cours du chargement dans le Tableau 3. Le premier point qui apparaît sur la Figure 6 est la dépendance des déformations vis-à-vis du chemin de contrainte suivi. La comparaison des essais Ch1 et Hu4 où le chargement mécanique a été effectué sous une succion de 8,5 MPa (Figure 6a) montre que la valeur de l’indice des vides dépend du chemin de contrainte suivi pour le même état de contrainte (succion de 8,5 MPa et 
contrainte verticale nette de $1200 \mathrm{kPa}$ ). Ce résultat est intéressant car il marque une différence importante entre les sols gonflants et non gonflants. Pour ces derniers en effet, les déformations sont indépendantes du chemin de contrainte suivi si la succion n’est pas augmentée au cours d’un essai (e.g. Alonso et al. 1990). Cela signifie que si un matériau non gonflant avait été employé, l’indice des vides aurait été le même dans les deux essais pour cet état de contrainte (succion de 8,5 MPa et contrainte verticale nette de $1200 \mathrm{kPa}$ ). Cette remarque va dans le sens des observations réalisées par Lloret et al. (2003) sur un autre sol gonflant. Cette tendance apparaît cependant moins marquée pour les autres essais où le chargement mécanique a été effectué sous une succion nulle (Figure 6b). Le second point mis en évidence par ces essais est que les chemins de contrainte suivis n’ont pas influencé de manière significative la valeur de la pente $\lambda(\mathrm{s})$ (Tableau 3). Dans le cas des essais présentés, le paramètre qui affecte le plus la pente $\lambda(s)$ est la succion imposée, le sol étant significativement moins compressible sous une succion de 8,5 MPa que sous une succion nulle. Il est donc possible de conclure que, dans les conditions des essais présentés dans cette partie, la pente $\lambda(\mathrm{s})$ ne dépend pas du chemin de contrainte, contrairement à l'indice des vides.

Les résultats des essais Hu1 et $\mathrm{V}_{c}$ peuvent être comparés. En effet, dans le cadre du modèle de Barcelone pour sols gonflants BExM (Barcelona Expansive Model, Gens et Alonso 1992 ; Alonso et al. 1999), la pression de gonflement mesurée par la méthode à volume constant correspond à la pression de préconsolidation, $\mathrm{p}_{0}(\mathrm{~s})$ sous une succion nulle. La pression de préconsolidation dans le cas de l'étude des sols non saturés dépend à la fois de la contrainte maximale subie par le sol mais aussi des sollicitations hydriques qu'il a subies. Il est donc nécessaire de parler de pression de préconsolidation apparente dans le cas de l'étude des sols non saturés. La valeur de cette pression de préconsolidation apparente, $\mathrm{p}_{0}(\mathrm{~s})$, sous succion nulle a été évaluée à partir du résultat de l'essai Hu1. Elle est comprise entre 55 et $60 \mathrm{kPa}$. Il 
est intéressant de noter que cette valeur est sensiblement égale à la pression de gonflement mesurée lors de l'essai $V_{c}$ (Figure 3). On peut aussi remarquer qu'il n’y a pas d'effondrement si la charge appliquée à une éprouvette pendant l’humidification est inférieure à cette valeur. Ces observations vont donc dans le sens de cette hypothèse formulée dans le cadre du modèle de Barcelone pour sols gonflants.

\section{INFLUENCE D’UNE SOLLICITATION HYDRIQUE CYCLIQUE SUR LE COMPORTEMENT MÉCANIQUE}

Une série d’essais a été entreprise pour caractériser l’influence de cycles hydriques conduits dans la gamme des succions comprises entre 8,5 et 40 MPa sur le comportement mécanique du matériau gonflant compacté étudié.

\section{Cas d'une contrainte appliquée de 10 kPa durant le cycle hydrique}

Les essais Cy1 et Cy2 commencent par un cycle hydrique réalisé sous une contrainte verticale de $10 \mathrm{kPa}$. Dans le cas de l'essai Cy1, le cycle hydrique débute, de son état de succion initiale de $20 \mathrm{MPa}$ environ, par l'imposition d'une succion de 8,5 MPa, la succion étant ensuite ramenée à une valeur de 20,5 MPa. Dans l'essai Cy2, en début de cycle hydrique, l'éprouvette est soumise à une succion de 38,9 MPa puis à une succion de 20,5 MPa. Après le cycle hydrique, chaque éprouvette a été chargée mécaniquement sous la même succion imposée de

20,5 MPa (Figure 7). Les caractéristiques initiales et finales des éprouvettes sont reportées dans le Tableau 3. La variation de l'indice des vides en fonction du temps, déterminée à \pm 0.005 , au cours de chaque cycle hydrique est donnée sur la Figure 8 qui permet de voir qu'un cycle d'humidification/dessiccation (essai Cy1) provoque l'apparition de déformations irréversibles de type gonflement alors qu’un cycle de dessiccation/humidification (essai Cy2) induit des déformations irréversibles de type retrait. Ces données confirment l’influence du chemin de contrainte sur l’indice des vides mise en évidence avec les essais précédents. 
Afin d'évaluer l'influence respective de la succion et de chaque cycle hydrique sur le comportement mécanique, deux essais additionnels ont été entrepris. Il s’agit des essais Ch2 et Ch3 qui correspondent à un chargement mécanique sous une succion de 20,5 et de 38.9 MPa respectivement (Figure 7). Les courbes de compressibilité obtenues dans ces essais sont reportées sur la Figure 9 et les paramètres mécaniques sont regroupés dans le Tableau 3. La comparaison des essais Cy1 et Ch2 montre que le cycle d'humidification/dessiccation n'a pas affecté de manière significative la valeur de la pente $\lambda$ (s) qui est identique dans les deux essais. En revanche, il apparaît que l'imposition du cycle de dessiccation/humidification de l'essai Cy2 a induit une réduction significative de la pente $\lambda(\mathrm{s})$. Le chargement mécanique étant effectué sous la même succion dans les deux cas, seule la prise en compte des différences de chemin de contrainte entre les essais Cy2 et Ch2 permet d'expliquer cette observation. De plus, la valeur de la pente $\lambda($ s) déterminée dans l'essai Cy2 est intermédiaire entre les valeurs de la pente $\lambda(\mathrm{s})$ obtenues dans les essais Ch2 et Ch3. La pente $\lambda$ (s) dépend donc du chemin de contrainte et de la succion sous laquelle le chargement est effectué. Il est intéressant de noter que seul le cycle de dessiccation/humidification semble avoir influencé la pente $\lambda(\mathrm{s})$. Afin de compléter ces observations, des essais supplémentaires comprenant un cycle hydrique sous une contrainte mécanique plus importante ont été réalisés.

\section{Cas d'une contrainte appliquée de $800 \mathrm{kPa}$ lors du cycle hydrique}

Dans l'essai Cy3 l'éprouvette a été chargée jusqu’à une contrainte verticale de 800 kPa sous une succion constante de 20,5 MPa (Figure 7). Ensuite, sa succion a été diminuée jusqu’à 8,5 $\mathrm{MPa}$ puis ramenée à 20,5 $\mathrm{MPa}$, la contrainte verticale étant maintenue à $800 \mathrm{kPa}$. Le chargement mécanique a ensuite été poursuivi sous une succion de 20,5 MPa. Le résultat de cet essai est reporté sur la Figure 10 et les caractéristiques de l'éprouvette dans le Tableau 3. Les points représentés sur la figure 10a donnent les valeurs de l'indice des vides lorsque l'équilibre est atteint suite à l'imposition d'une succion donnée. La réduction de succion de 
20,5 à 8,5 MPa a provoqué un effondrement de l'éprouvette, ce qui est similaire à ce qui avait été observé dans l'essai Hu4 (Figure 5) dans les mêmes conditions. Le retour vers la succion initiale s'est accompagné d’une rétraction importante de l'éprouvette. Le cycle d’humidification/dessiccation a donc généré des déformations irréversibles. La comparaison des essais Cy1 et Cy3 montre que le type de déformations irréversibles induit par un cycle hydrique dépend de la contrainte verticale appliquée pendant le cycle hydrique. Ces conclusions sont similaires à celles avancées par Alonso et al. (1995) et par Kato et Kawai (2000). En revanche, il n'est pas possible de conclure quant à l’influence de ce cycle sur la pente $\lambda(s)$ avec cet essai car l'éprouvette se comporte de manière élastique durant la fin du chargement/déchargement mécanique.

Dans le cas de l'essai Cy4 (Figure 7), l'éprouvette a été chargée mécaniquement jusqu’à 800 kPa sous une succion imposée de 20,5 MPa dans un œdomètre à solutions salines. Un cycle hydrique divisé en deux phases a ensuite été entrepris, la contrainte verticale étant maintenue à une valeur de $800 \mathrm{kPa}$ tout au long du cycle. La première phase correspond à une dessiccation jusqu'à une succion de 38,9 MPa suivie d'un retour à la succion de 20,5 MPa. Lors de la deuxième phase, une succion de 8,5 MPa a été imposée. Ensuite, l’éprouvette a été chargée et déchargée mécaniquement, sous une succion imposée de 8,5 MPa. Les caractéristiques de l'éprouvette sont reportées dans le Tableau 3. La Figure 11 donne la variation de l'indice des vides durant cet essai (les points sur la figure 11a représentent l'indice des vides lorsque l'équilibre est atteint suite à l’imposition d'une succion donnée). L'augmentation de succion de 20,5 à 38,9 MPa a provoqué un retrait de l'éprouvette. Le retour à la succion de 20,5 MPa s’est accompagné d’un léger gonflement du matériau. La première partie du cycle hydrique a donc induit un retrait irréversible du matériau. Le passage d'une succion imposée de 20,5 à 8,5 MPa s'accompagne d'un effondrement de l'éprouvette. Le comportement sur cette partie du chemin de contrainte est semblable à la phase 
d'effondrement conduit dans l'essai Hu4 qui correspondait à un essai d'humidification sous une contrainte de 800 kPa (cf. Figures 4 et 5). La dessiccation imposée dans le cas de l'essai Cy4 n’a donc pas affecté la tendance à l'effondrement du matériau, la réduction de l'indice des vides étant cependant plus importante dans le cas de l'essai Hu4. La courbe de compressibilité de l'essai Cy4 est reportée avec celles des essais Ch1 et Hu4 sur la Figure 12. Cette Figure met de nouveau en évidence l'influence du chemin de contrainte sur les déformations subies par le matériau testé. De plus, elle permet la comparaison des valeurs des pentes $\lambda$ (s) déterminées dans chacun de ces essais qui incluent tous un chargement mécanique sous une succion de $8,5 \mathrm{MPa}$ (Tableau 3). Il apparaît ainsi que la pente $\lambda$ (s) est significativement plus faible dans le cas de l'essai Cy4 que dans les essais Ch1 et Hu4. La valeur de $\lambda(\mathrm{s})$ dans cet essai est intermédiaire entre les valeurs de $\lambda(\mathrm{s})$ déterminées lors des essais Ch3 et Ch1.

Il est donc possible de conclure que dans le cas de ces essais, la pente de compression plastique dépend à la fois de la succion imposée au cours du chargement mécanique mais aussi du chemin de contrainte suivi. Il est intéressant de noter que seule une dessiccation semble affecter la valeur de la pente $\lambda(\mathrm{s})$ puisque dans le cas de l'essai Hu4, la pente $\lambda(\mathrm{s})$ est identique à celle déterminée lors de l'essai Ch1. Ces observations sont assez différentes de celles de Sharma (1998) cité par Gallipoli et al. (2003) qui ont obtenu une baisse de la pente $\lambda$ (s) suite à l'imposition d'un cycle d'humidification/dessiccation. L'influence de la texture du sol mise en évidence par Wheeler et Sivakumar (2000) et Cuisinier et Masrouri (2004a) devrait permettre une meilleure caractérisation de ces phénomènes.

\section{CONCLUSION}

Ce travail s’inscrit dans le cadre général de l'étude l’influence de sollicitations complexes sur le comportement hydromécanique d’un sol gonflant compacté qui présente un grand intérêt 
pour toutes les applications en géotechnique de l'environnement dans lesquelles les matériaux argileux compactés sont employés.

Les résultats obtenus ont permis de montrer que l'effondrement est un phénomène progressif dont l'ampleur dépend fortement de la contrainte verticale appliquée lors de l'humidification. De plus, pour un sol gonflant compacté, les déformations dépendent du chemin de contrainte suivi, quel que soit le type de sollicitation hydrique imposée (dessiccation ou humidification). Il est aussi intéressant de noter qu'un cycle hydrique d'amplitude limitée peut induire des déformations irréversibles d'une amplitude significative. Ces résultats vont dans le sens des quelques résultats existants obtenus par des essais à succion contrôlée sur ces phénomènes.

Les différents essais entrepris ont aussi permis de montrer que, sous certaines conditions, la pente de compression plastique $\lambda(\mathrm{s})$ dépendait du chemin de contrainte suivi. Cette observation s’est trouvée vérifiée dans le cas des essais comportant un cycle hydrique incluant l'imposition d'une succion supérieure à la succion initiale des éprouvettes (essais Cy2 et Cy4). En effet, dans le cas des autres essais comportant des sollicitations hydriques mais sans imposition d'une succion supérieure à la succion initiale des éprouvettes, la pente $\lambda(s)$ est apparue indépendante du chemin de contrainte. Dans ces essais, seule la succion appliquée lors du chargement est apparue jouer un rôle significatif sur la valeur de ce paramètre. La pente $\lambda(s)$ est donc une combinaison de la succion sous laquelle s'effectue le chargement mécanique, mais aussi de «l’histoire » du matériau. 


\section{RÉFÉRENCES}

Alonso, E.E., Gens, A., et Josa, A. 1990. A constitutive model for partially saturated soils. Géotechnique, 40 : 405-430.

Alonso, E.E., Lloret, A., Gens, A., et Yang, D.Q. 1995. Experimental behaviour of highly expansive double-structure clay. Proceedings of the $1^{\text {st }}$ International Conference on Unsaturated Soils, Paris, vol. 1, pp. 11-16.

Alonso, E.E., Vaunat, J., et Gens, A. 1999. Modelling the mechanical behaviour of expansive clays. Engineering Geology, 54 : 173-183.

ASTM D 5298 - 94, 1995a. Measurement of soil potential (suction) using filter paper. Vol. 4.09, pp. 154-159.

ASTM D 4546 - 90, 1995b. One-dimensional swell or settlement potential of cohesive soils. Vol. 4.08,p p. 693-699.

Barden, L., Madedor, A.O., et Sides, G.R. 1969. Volume change characteristics of unsaturated clays. ASCE Journal of Soil Mechanics and Foundation Analysis, 96: 33-51.

Barrera, N., Romero, E., Lloret, A., et Gens, A. 2002. Collapse tests on isotropic and anisotropic compacted soils. Proceedings of the International Workshop on Unsaturated Soils, Trento, Italie, pp. 33-46.

Brackley, I.J. 1973. Swell pressure and free swell in compacted clay. Proceedings of the $3^{\text {rd }}$ International Conference on Expansive Soils, Haifa, vol. 1, pp. 169-176.

Chapman, N., et Tassoni, E. 1985. Feasibility studies for a radioactive waste repository in a deep clay formation. Office for official publications of the European communities (Luxembourg), Rapport n EUR 10061 EN, 200 p.

Chen, F.H. 1975. Foundations on expansive soils. Developments in Geotechnical Engineering, vol. 12, Elsevier (Amsterdam). 280 p. 
Coleman J.D. 1962. Stress-strain relations for partly saturated soils. Géotechnique, vol. 12, pp. 348-350.

Collins, K., et McGown, A. 1974. The form and function of microfabric features in a variety of natural soils. Géotechnique, 24 : 223-254.

Cui, Y.J., Loiseau, C., et Delage, P. 2002. Microstructure changes of a confined swelling soil due to suction controlled hydration. Proceedings of the $3^{\text {rd }}$ International Conference on Unsaturated Soils, Recife, Brazil, vol. 2, pp. 593-598.

Cuisinier, O. 2002. Comportement hydromécanique des sols gonflants compactés. Thèse de Doctorat, Institut National Polytechnique de Lorraine, Nancy, France, 160 p.

Cuisinier, O., et Masrouri, F. 2003. Comportement hydromécanique d'un sol gonflant sous très fortes succions. Comptes Rendus Mécanique, 333 : 203-210.

Cuisinier, O. et Masrouri, F. 2004a. Testing the hydromechanical behavior of a compacted swelling soil. ASTM Geotechnical Testing Journal, 27 : 598-606.

Cuisinier, O. et Masrouri, F. 2004b. Structure et comportement mécanique d'un sol gonflant non saturé. Revue Française de Géotechnique, 109 : 47-56.

Day, R.W. 1994. Swell-shrink behavior of compacted clay. Journal of Geotechnical Engineering, 120 : 618-623.

Delage, P., Howat, M.D., et Cui, Y.J. 1998. The relationship between suction and the swelling properties in a heavily compacted swelling clay. Engineering Geology, 50 : 31-48.

Dif, A.E., et Bluemel, W.F. 1991. Expansive soils under cyclic drying and wetting. Geotechnical Testing Journal, 14 : 96-102.

Fredlund D.G., Morgenstern N.R. 1977. Stress state variables for unsaturated soils. Journal of Geotechnical Engineering, vol. 103, pp. 447-466. 
Gallipoli, D., Gens, A., Sharma, R., et Vaunat, J. 2003. An elasto-plastic model for unsaturated soil incorporating the effects of suction and degree of saturation on mechanical behaviour. Géotechnique, 53 : 123-135.

Gens, A., et Alonso, E.E. 1992. A framework for the behaviour of unsaturated expansive clays. Canadian Geotechnical Journal, 29 : 1013-1032.

Gens, A., et Olivella, S. 2001. Clay barriers in radioactive waste disposal. Revue Française de Génie Civil, 5-6 : 845-856.

Guiras-Skandaji, H. 1996. Déformabilité des sols argileux non saturés : étude expérimentale et application à la modélisation. Thèse de Doctorat, Institut National Polytechnique de Lorraine, Nancy, France, 315 p.

Kato, S., et Kawai, K. 2000. Deformation characteristics of a compacted clay in collapse under isotropic and triaxial stress state. Soils and Foundations, 40 : 75-90.

Koch, D. 2002. Bentonites as a basic material for technical base liners and site encapsulation cut-off walls. Applied Clay Science, 21 : 1-11.

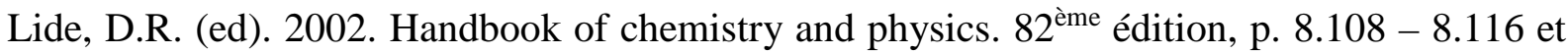
$15.25-15.26$

Lloret, A., Villar, M.V., Sanchez, M., Gens, A., Pintado, X. et, Alonso, E.E. 2003. Mechanical behaviour of heavily compacted bentonite under high suction changes. Géotechnique, 53 : 27-40.

Matyas E.L., Radhakrishna H.S. 1968. Volume change characteristics of partially saturated soils. Géotechnique, vol. 18, pp. 432-448. Osipov, V.I., Nguen Ngok Bik, et Rumjantseva, N.A. 1987. Cyclic swelling of clays. Applied Clay Science, 2 : 363-374.

Push, R. 1982. Mineral-water interactions and their influence on the physical behaviour of highly compacted Na-bentonite. Canadian Geotechnical Journal, 19 : 381-387. 
Philipponat, G. 1991. Retrait-gonflement des argiles, proposition de méthodologie. Revue Française de Géotechnique, 57 : 5-22.

Romero, E., Gens, A., et Lloret, A. 1999. Water permeability, water retention and microstructure of unsaturated Boom clay. Engineering Geology, 54 : 117-127.

Tessier, D. 1984. Étude expérimentale de l'organisation des matériaux argileux - Hydratation, gonflement et structuration au cours de la dessiccation et de la réhumectation. Thèse de doctorat, Université Paris VII, France, 362 p.

Vandangeon, P. 1992. Exemples de sinistres en région parisienne. Revue Française de Géotechnique, 58 : 892-898.

Villar, M.V. 1999. Investigation of the behaviour of bentonite by means of suction-controlled oedometer tests. Engineering Geology, 54 : 67-73.

Villar, M. V. et Martín, P. L., 1996. Suction-controlled oedometer tests in montmorillonite clay: preliminary results. Engineering Geology of Waste Disposal, Geological Society of Engineering Geology, Special Publication, 11 : 309-312.

Wheeler, S.J., et Sivakumar, V., 2000. Influence of compaction procedure on the mechanical behaviour of an unsaturated compacted clay. Part 2 : shearing and constitutive modelling. Géotechnique, 50 : 369-376.

Williams, J., et Shaykewich, C.F. 1969. An evaluation of polyethylene glycol (PEG) 6000 and PEG 20000 in the osmotic control of soil water matric potential. Canadian Journal of Soil Science, 49 : 397-401.

Zur, B., 1966. Osmotic control of the matric soil-water potential : I. Soil water system. Soil Science, 102 : 394-398. 


\section{LÉGENDE DES FIGURES.}

Figure 1. Principe d'un œdomètre à succion contrôlée par la méthode osmotique d'après Cuisinier et Masrouri (2003b)..

Figure 2. Principe d'un œdomètre à succion contrôlée par la méthode des solutions salines d'après Cuisinier et Masrouri (2003b)

Figure 3. Variation de la pression exercée par le matériau au cours d'une humidification à volume constant (essai Vc).

Figure 4. Chemins de contrainte suivis pour étudier l'influence d'une sollicitation hydrique monotone sur le comportement mécanique.

Figure 5. Influence de la charge verticale appliquée lors de l’humidification sur les variations de volume.

Figure 6. Influence d'une humidification sous charge sur le comportement mécanique d'un sol gonflant : (a) cas où le chargement mécanique après l'humidification est effectué sous une succion de $8,5 \mathrm{MPa}$; (b) cas où le chargement mécanique après l’humidification est effectué sous une succion nulle.

Figure 7. Chemins de contrainte des essais suivis pour étudier l'influence d'une sollicitation hydrique complexe sur le comportement mécanique.

Figure 8. Variations de l'indice des vides au cours d'un cycle hydrique conduit sous une contrainte verticale de $10 \mathrm{kPa}$ ( $\mathrm{D}$ : dessiccation ; $\mathrm{H}$ : humidification). 
Figure 9. Comparaison des essais Cy1 et Cy2 avec les essais Ch1, Ch2 et Ch3 pour évaluer l'influence d'un cycle hydrique conduit sous une faible charge verticale (10 kPa) sur la compressibilité.

Figure 10. Résultat de l'essai Cy3 : (a) variation de l'indice des vides au cours du cycle hydrique réalisé sous une contrainte verticale de $800 \mathrm{kPa}$; (b) courbe de compressibilité.

Figure 11. Résultat de l'essai Cy4 : (a) variation de l'indice des vides au cours du cycle hydrique réalisé sous une contrainte verticale de $800 \mathrm{kPa}$; (b) courbe de compressibilité.

Figure 12. Influence d'un cycle hydrique conduit sous une forte charge verticale (800 kPa) sur la compressibilité (chargement final conduit sous une succion de 8,5 MPa dans ces essais). 
TABLEAU 1. Solutions salines employées et succions imposées correspondantes.

\begin{tabular}{cccc}
\hline Sel & $\begin{array}{c}\text { Solubilité }^{*}+ \\
\text { (g de sel par litre } \\
\text { d'eau) }\end{array}$ & $\begin{array}{c}\text { Humidité relative }{ }^{*}{ }^{\dagger} \\
(\%)\end{array}$ & $\begin{array}{c}\text { Succion imposée } \\
(\mathrm{MPa})\end{array}$ \\
\hline $\mathrm{LiCl}, \mathrm{x} \mathrm{H}_{2} \mathrm{O}$ & 827 & 12 & 287,9 \\
$\mathrm{MgCl}_{2}, 6 \mathrm{H}_{2} \mathrm{O}$ & 567 & 33 & 150,6 \\
$\mathrm{Mg}\left(\mathrm{NO}_{3}\right)_{2}, 6 \mathrm{H}_{2} \mathrm{O}$ & 689 & 54 & 83,6 \\
$\mathrm{NaCl}$ & 360 & 75 & 38,9 \\
$\mathrm{KCl}$ & 341 & 86 & 20,5 \\
$\mathrm{KNO}_{3}$ & 320 & 94 & 8,5 \\
\hline
\end{tabular}

* : les valeurs de ce Tableau sont données pour une température de $20 \pm 0,1^{\circ} \mathrm{C}$.

$\dagger$ : Lide (2002), pp. $8.108-8.116$.

Cuisinier et Masrouri 
TABLEAU 2. Propriétés géotechniques des matériaux employés.

\begin{tabular}{ccccc}
\hline Matériau & $\mathrm{W}_{\mathrm{L}}$ & $\mathrm{Ip}$ & $\rho_{\mathrm{s}}$ & Classe \\
& $(\%)$ & $(\%)$ & $\left(\mathrm{kg} \cdot \mathrm{m}^{-3}\right)$ & USCS \\
\hline Limon & 42 & 5 & 2700 & $\mathrm{ML}$ \\
Bentonite & 117 & 31 & 2600 & $\mathrm{MH}$ \\
Mélange & 87 & 21 & 2600 & $\mathrm{MH}$ \\
\hline plasticité. & & & &
\end{tabular}

* : Ip est l’indice de plasticité.

Cuisinier et Masrouri 
TABLEAU 3. Caractéristiques générales des éprouvettes testées et valeurs de la pente de compression plastique $\lambda(\mathrm{s})$.

\begin{tabular}{lcccccc}
\hline Essai & $\mathrm{w}_{\mathrm{i}}$ & $\mathrm{\rho d}_{\mathrm{i}}$ & $\mathrm{Sr}_{\mathrm{i}}$ & $\mathrm{W}_{\mathrm{f}}$ & $\mathrm{Sr}_{\mathrm{f}}$ & $\lambda(\mathrm{s})$ \\
& $(\%)$ & $\left(\mathrm{kg} \cdot \mathrm{m}^{-3}\right)$ & $(\%)$ & $(\%)$ & $(\%)$ & $(-)$ \\
\hline $\mathrm{V}_{\mathrm{c}}$ & 14,3 & 1270 & 34,5 & 40,7 & 98,3 & $/$ \\
Hu1 & 14,6 & 1260 & 34,7 & 45,6 & 100,0 & 0,23 \\
Hu2 & 15,0 & 1270 & 36,2 & 32,9 & 100,0 & 0,23 \\
Hu3 & 15,0 & 1270 & 36,2 & 33,2 & 100,0 & 0,21 \\
Hu4 & 15,1 & 1270 & 36,4 & 16,6 & 61,2 & 0,30 \\
Ch1 & 15,0 & 1260 & 36,2 & 16,8 & 58,8 & 0,31 \\
Ch2 & 15,1 & 1270 & 36,4 & 14,9 & 48,8 & 0,27 \\
Ch3 & 15,1 & 1260 & 35,9 & 11,5 & 36,1 & 0,19 \\
Cy1 & 14,9 & 1260 & 35,4 & 14,3 & 38,3 & 0,29 \\
Cy2 & 14,7 & 1270 & 35,5 & 14,6 & 39,7 & 0,22 \\
Cy3 & 15,4 & 1280 & 37,7 & 15,0 & 42,8 & $/$ \\
Cy4 & 15,4 & 1290 & 38,3 & 16,6 & 49,3 & 0,21 \\
\hline & & & & & & \\
\hline
\end{tabular}

Cuisinier et Masrouri 


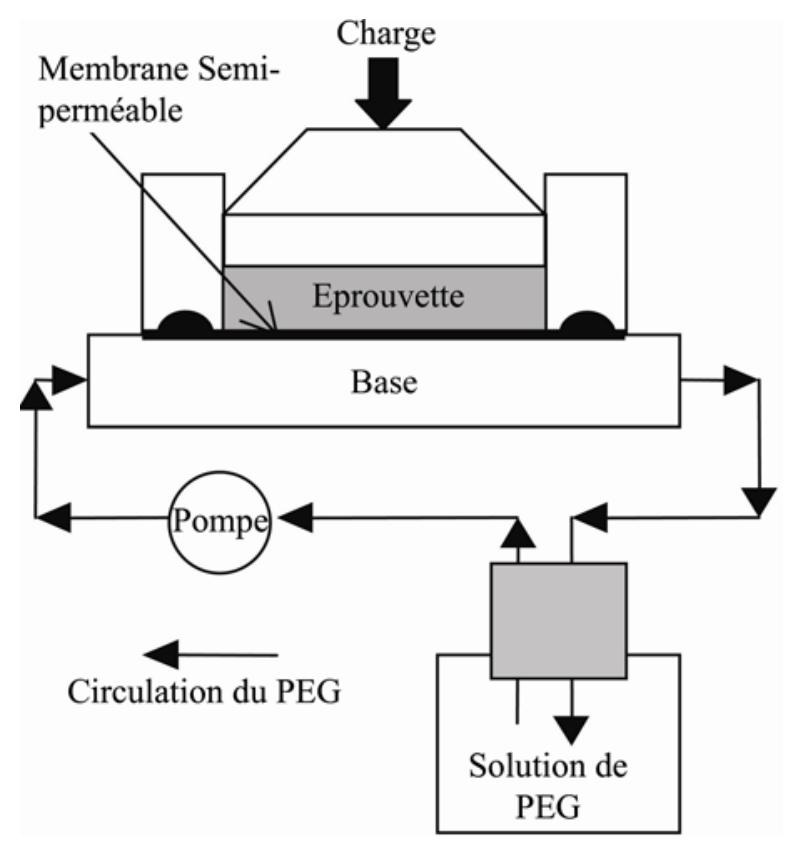

Figure 1. Principe d'un œdomètre à succion contrôlée par la méthode osmotique d'après Cuisinier et Masrouri (2003b).

Cuisinier et Masrouri 


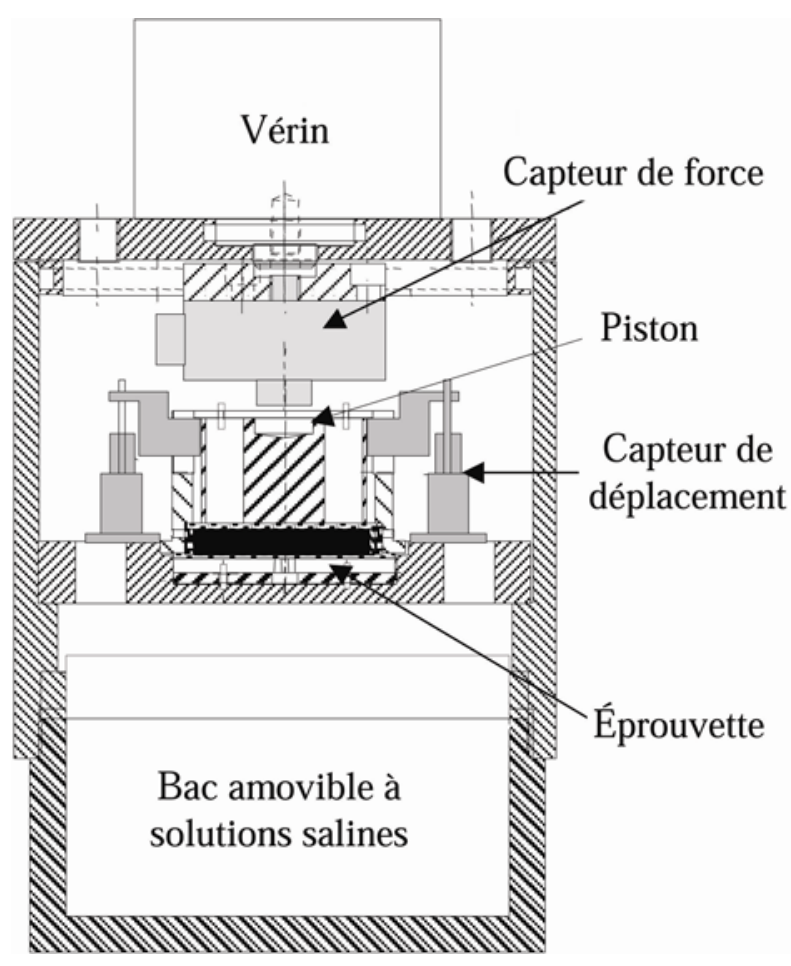

Figure 2. Principe d'un œdomètre à succion contrôlée par la méthode des solutions salines d'après Cuisinier et Masrouri (2003b).

Cuisinier et Masrouri. 


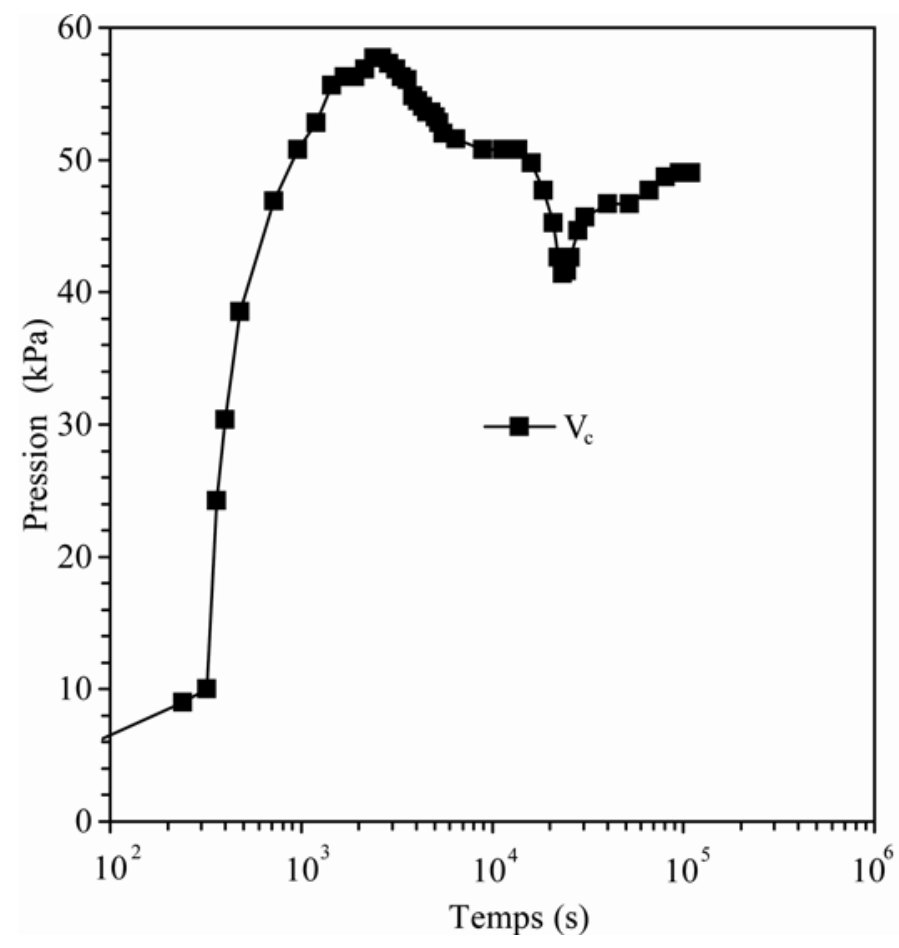

Figure 3. Variation de la pression de gonflement au cours d'une humidification à volume constant.

Cuisinier et Masrouri. 


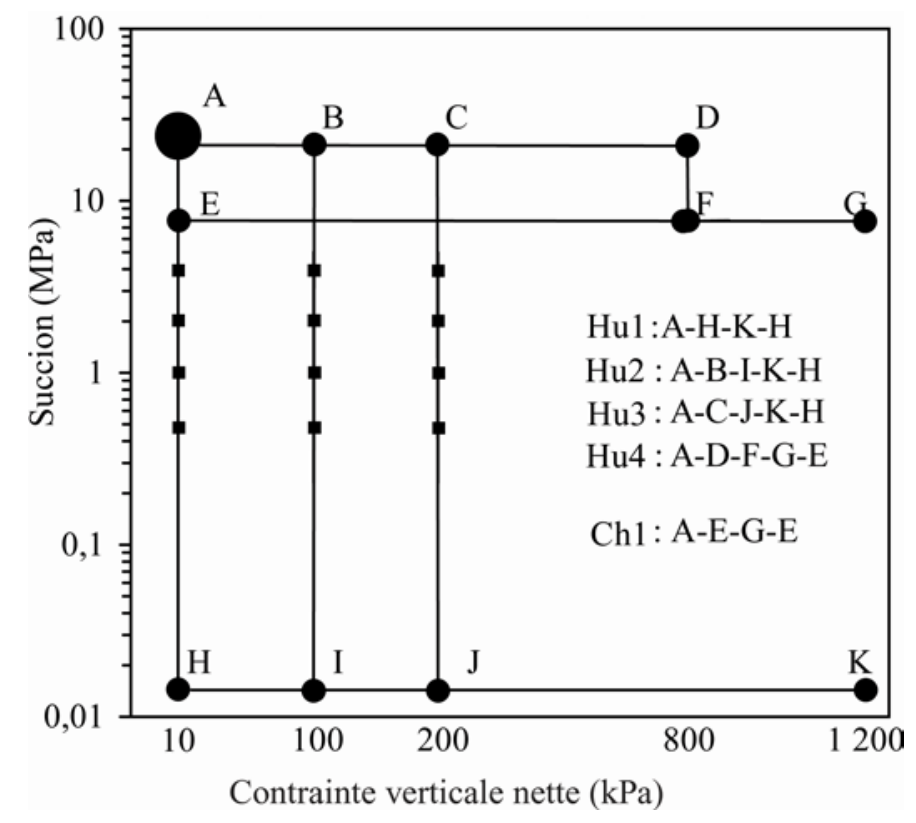

Figure 4. Chemins de contrainte suivis pour étudier l'influence d'une sollicitation hydrique monotone sur le comportement mécanique.

Cuisinier et Masrouri 


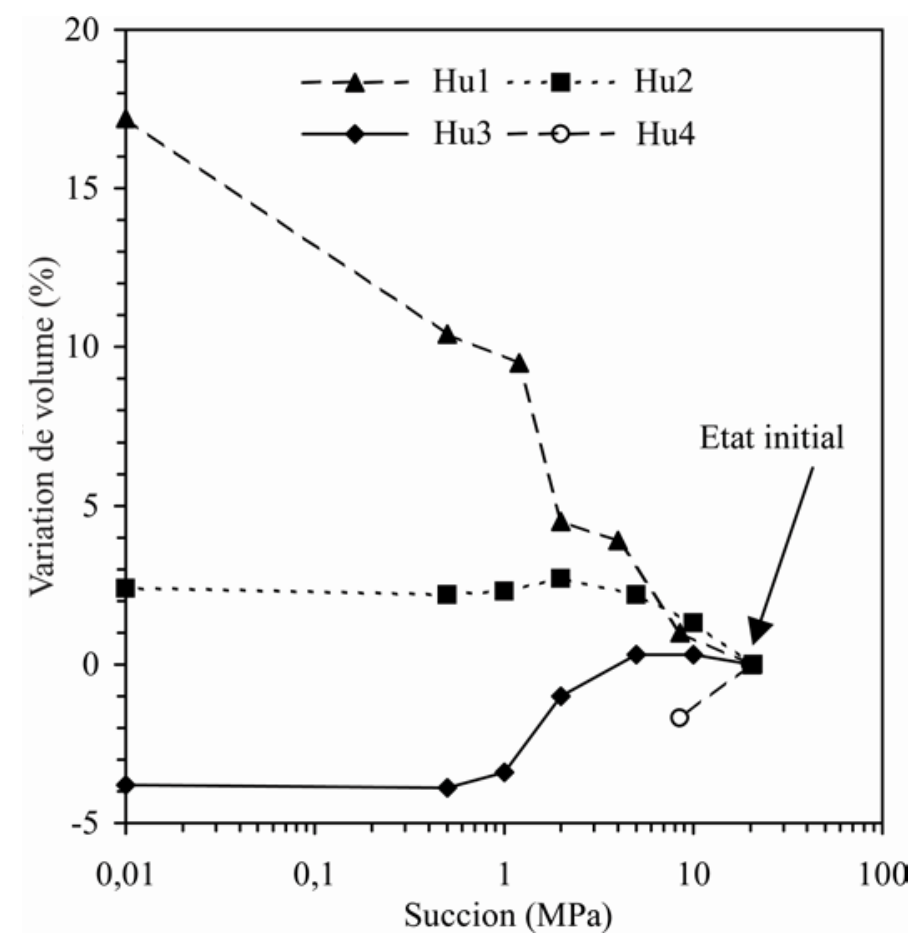

Figure 5. Influence de la charge verticale appliquée lors de l'humidification sur les variations de volume.

Cuisinier et Masrouri. 

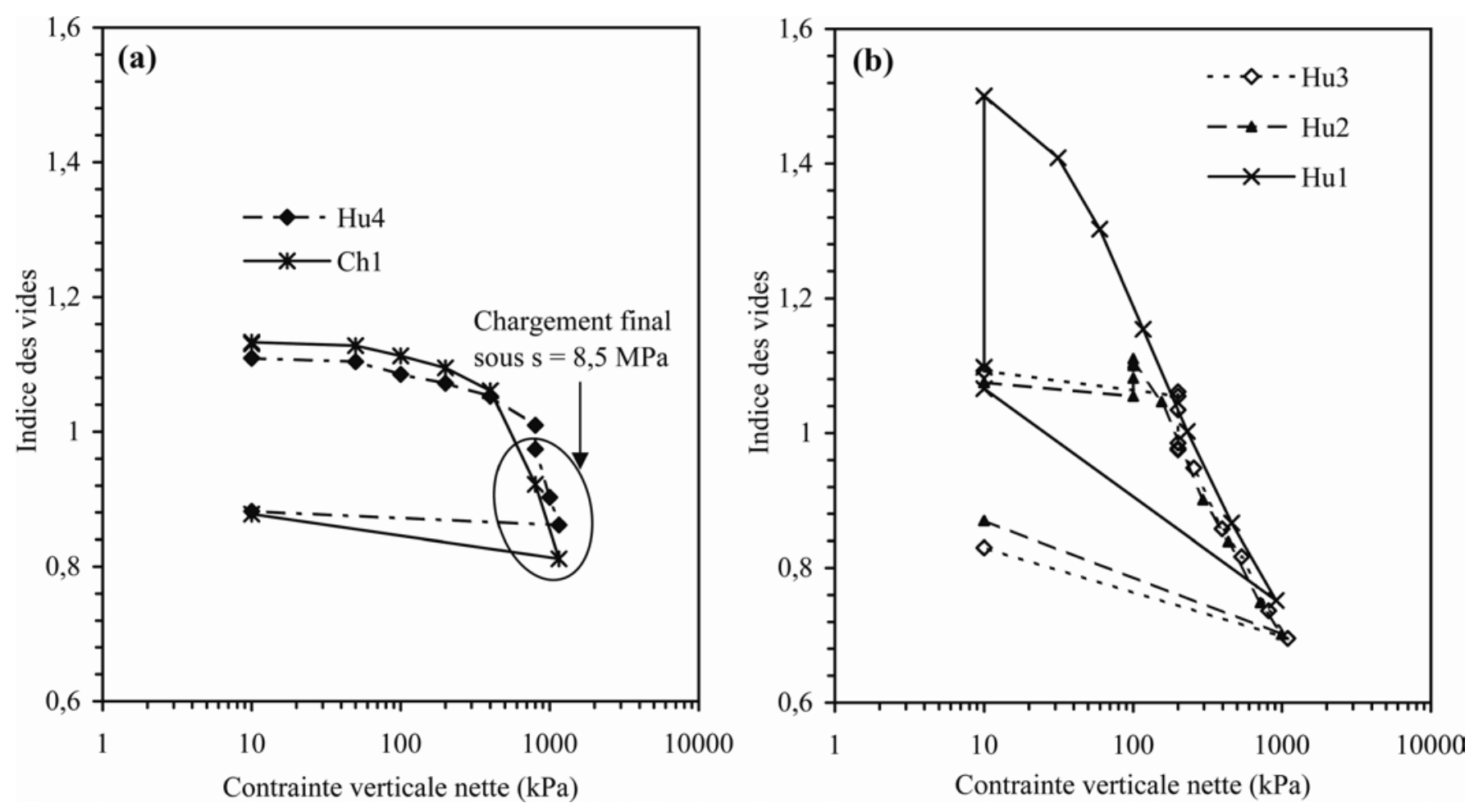

Figure 6. Influence d'une humidification sous charge sur le comportement mécanique d'un sol gonflant : (a) cas où le chargement mécanique après l'humidification est effectué sous une succion de 8,5 MPa ; (b) cas où le chargement mécanique après l'humidification est effectué sous une succion nulle.

Cuisinier et Masrouri. 


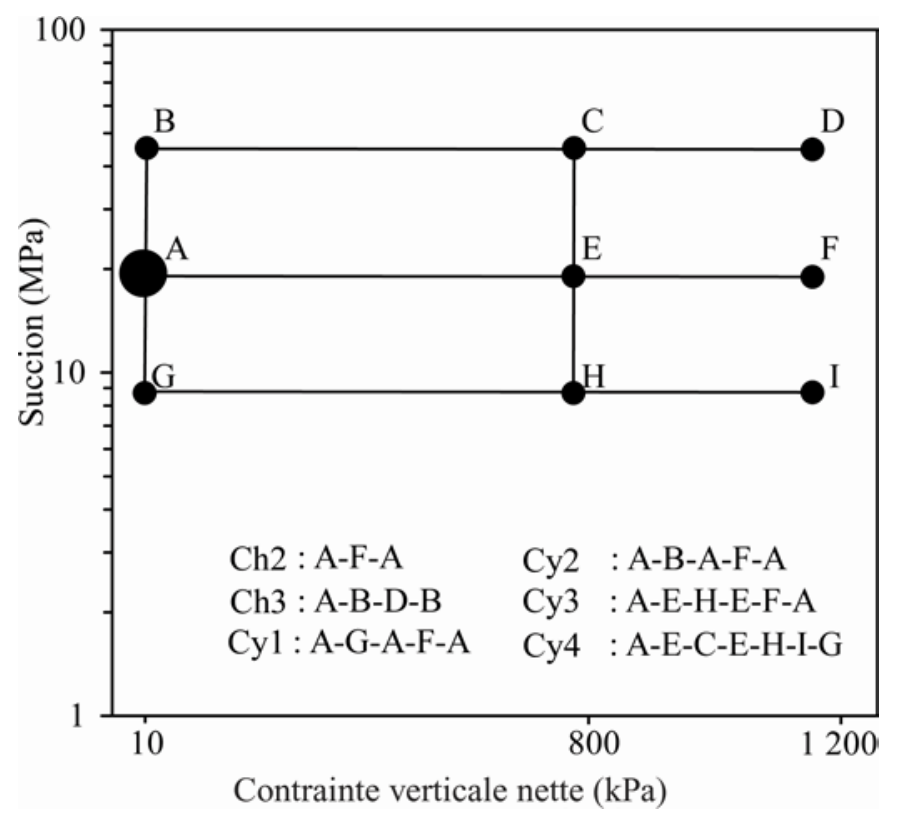

Figure 7. Chemins de contrainte des essais suivis pour étudier l'influence d'une sollicitation hydrique complexe sur le comportement mécanique.

Cuisinier et Masrouri. 


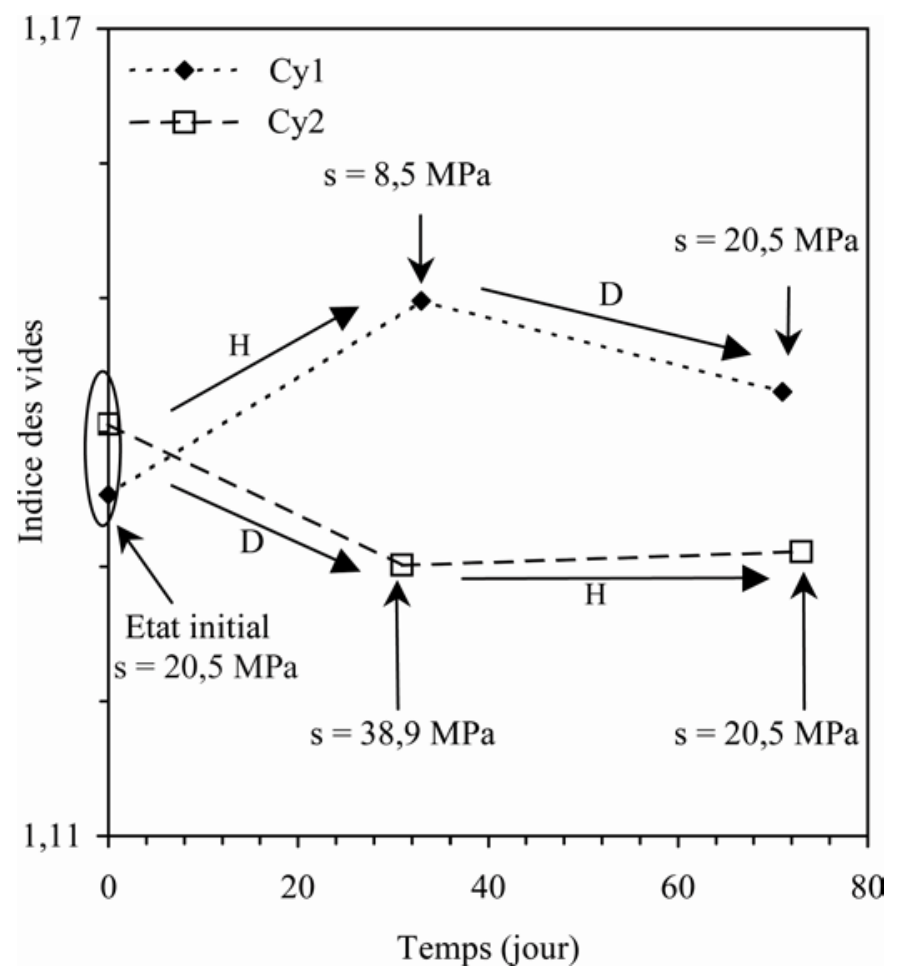

Figure 8. Variations de l'indice des vides au cours d'un cycle hydrique conduit sous une contrainte verticale de $10 \mathrm{kPa}$ (D : dessiccation ; $\mathrm{H}$ : humidification).

Cuisinier et Masrouri. 


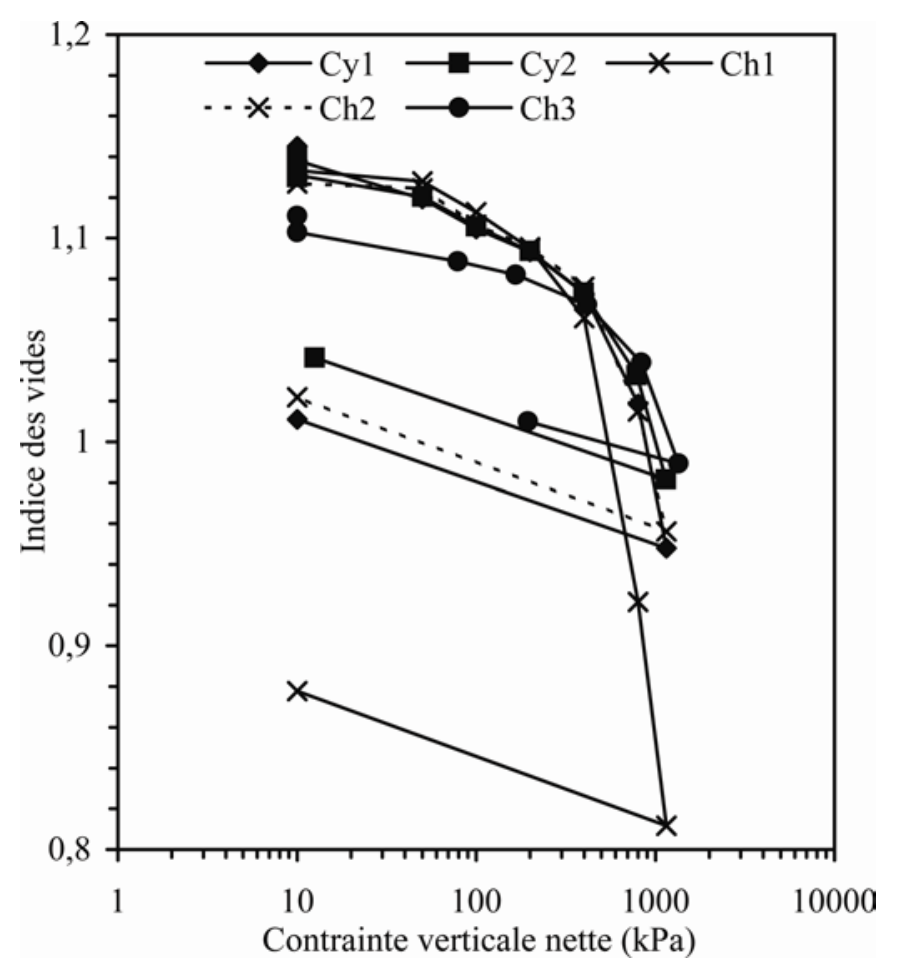

Figure 9. Comparaison des essais Cy1 et Cy2 avec les essais Ch1, Ch2 et Ch3 pour évaluer l'influence d'un cycle hydrique conduit sous une faible charge verticale (10 kPa) sur la compressibilité.

Cuisinier et Masrouri. 

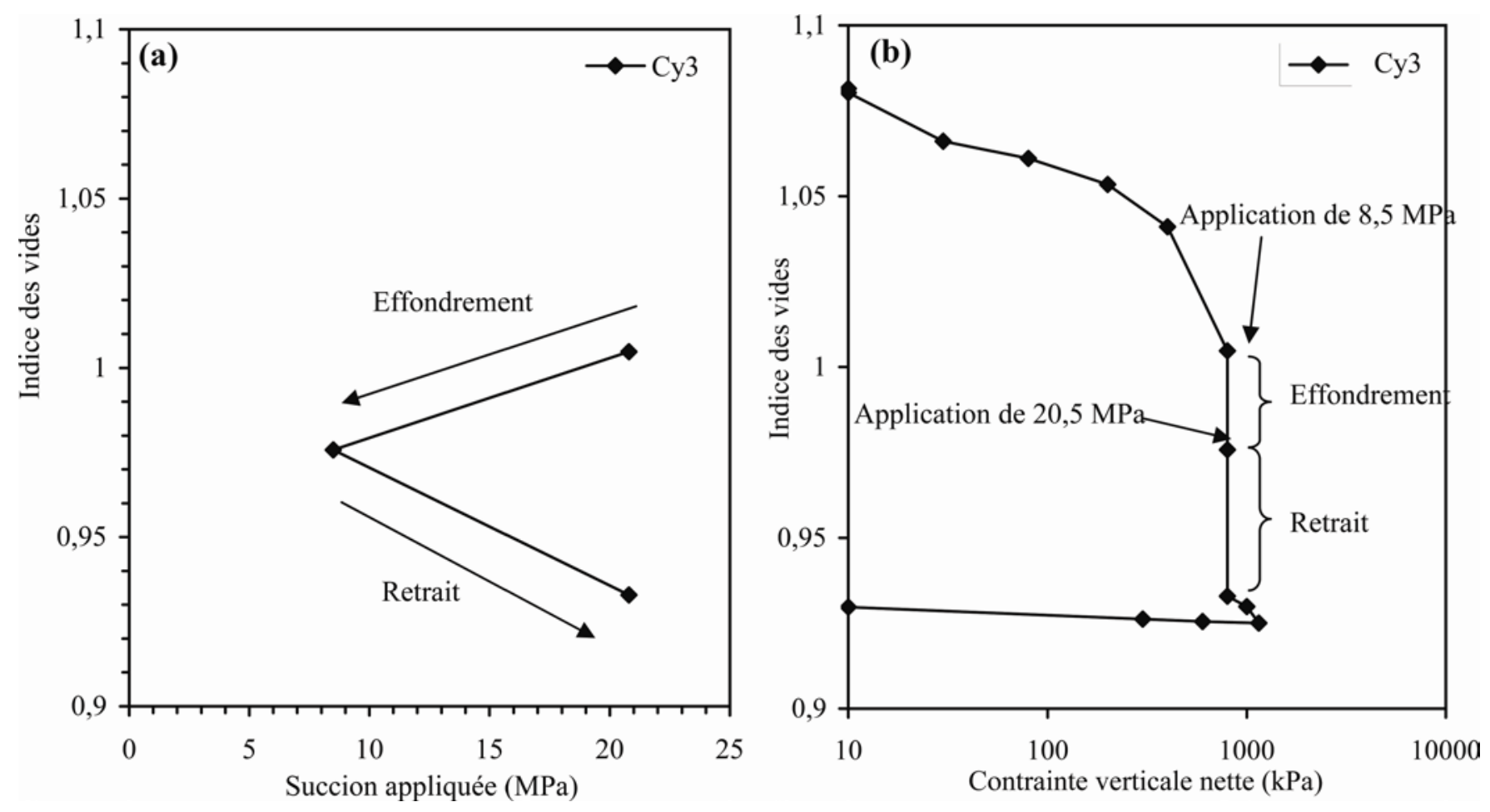

Figure 10. Résultat de l'essai Cy3 : (a) variation de l'indice des vides au cours du cycle hydrique réalisé sous une contrainte verticale de 800 kPa ; (b) courbe de compressibilité.

Cuisinier et Masrouri. 

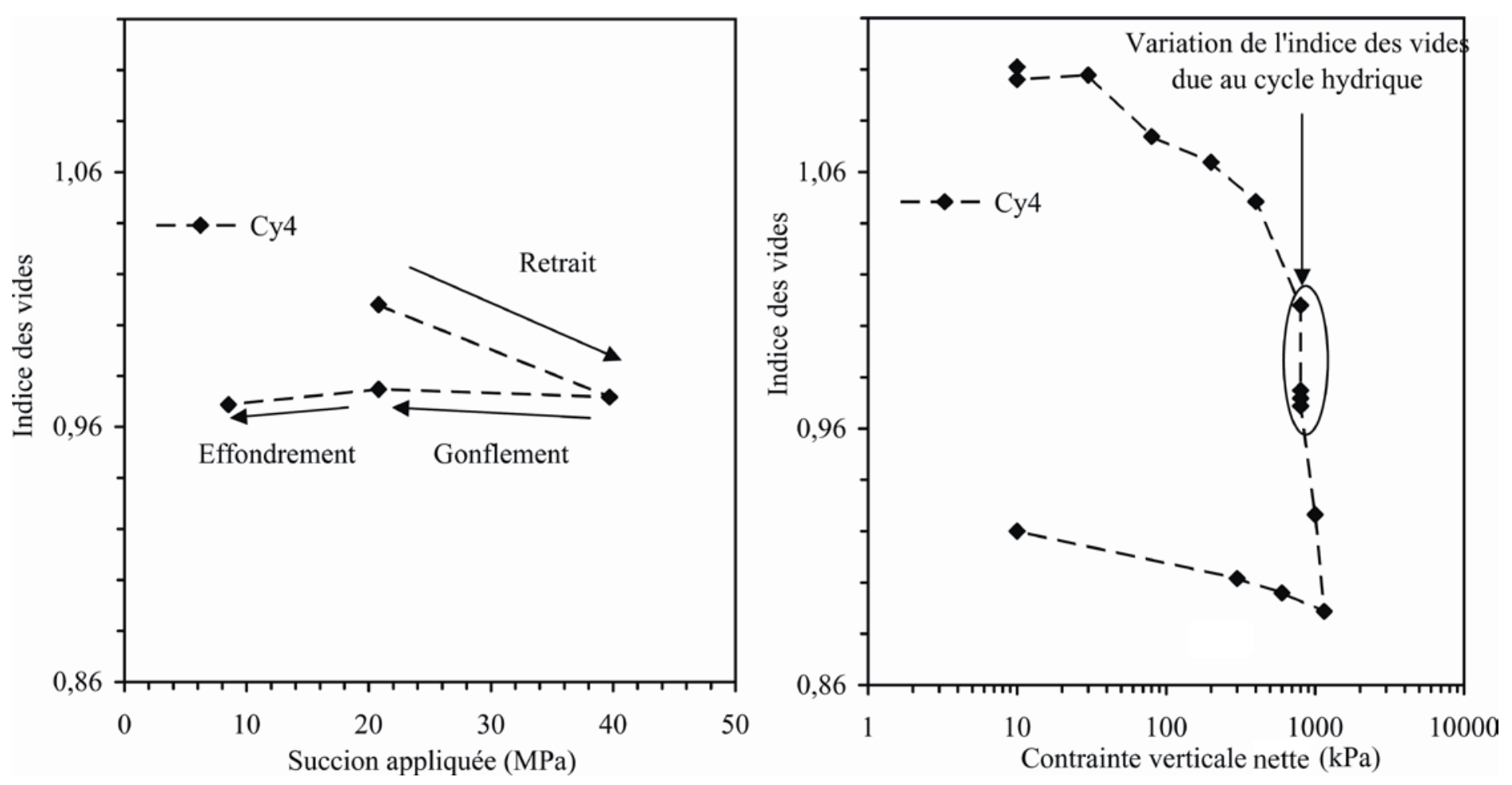

Figure 11. Résultat de l'essai Cy4 : (a) variation de l'indice des vides au cours du cycle hydrique réalisé sous une contrainte verticale de 800 kPa ; (b) courbe de compressibilité.

Cuisinier et Masrouri. 


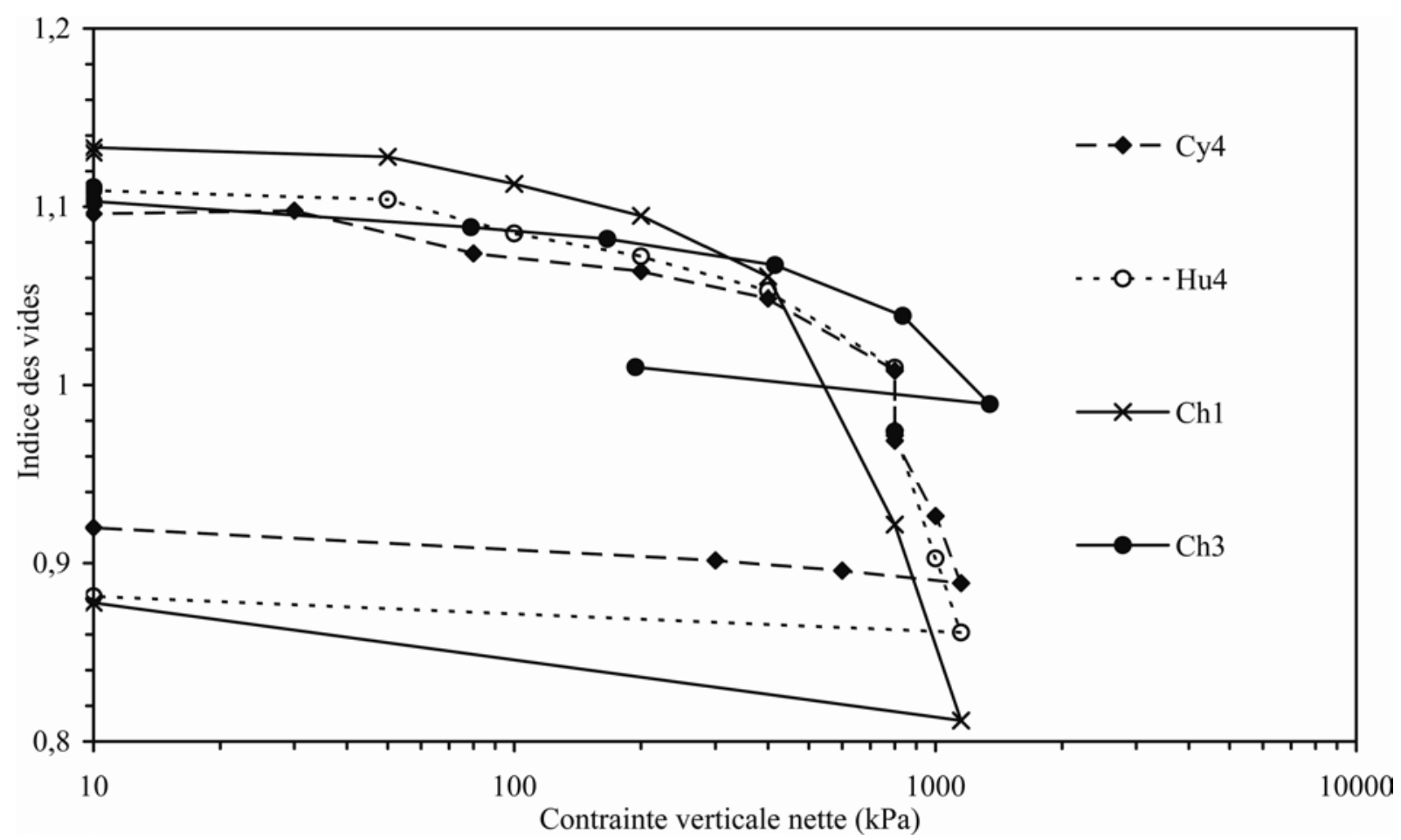

Figure 12. Influence d'un cycle hydrique conduit sous une forte charge verticale (800 kPa) sur la compressibilité (chargement final conduit sous une succion de 8,5 MPa dans ces essais).

Cuisinier et Masrouri. 